\title{
BMJ Open Developing a framework to incorporate real-world evidence in cancer drug funding decisions: the Canadian Real- world Evidence for Value of Cancer Drugs (CanREValue) collaboration
}

Kelvin Chan, ${ }^{1,2,3,4}$ Seungree Nam (1) ${ }^{2,3}$ Bill Evans, ${ }^{5}$ Claire de Oliveira, ${ }^{6}$ Alexandra Chambers, ${ }^{7}$ Scott Gavura, ${ }^{4}$ Jeffrey Hoch, ${ }^{8}$ Rebecca E Mercer, ${ }^{3,4}$ Wei Fang Dai, ${ }^{3,4}$ Jaclyn Beca, ${ }^{3,4}$ Mina Tadrous, ${ }^{9}$ Wanrudee Isaranuwatchai ${ }^{10,11}$

To cite: Chan K, Nam S, Evans B, et al. Developing a framework to incorporate realworld evidence in cancer drug funding decisions: the Canadian Real-world Evidence for Value of Cancer Drugs (CanREValue) collaboration. BMJ Open 2020;10:e032884. doi:10.1136/ bmjopen-2019-032884

- Prepublication history and additional material for this paper are available online. To view these files, please visit the journal online (http://dx.doi org/10.1136/bmjopen-2019032884).

Received 10 July 2019 Revised 14 November 2019 Accepted 05 December 2019

Check for updates

(C) Author(s) (or their employer(s)) 2020. Re-use permitted under CC BY-NC. No commercial re-use. See rights and permissions. Published by BMJ.

For numbered affiliations see end of article.

Correspondence to

Dr Kelvin Chan;

kelvin.chan@sunnybrook.ca

\section{ABSTRACT}

Background Oncology therapy is becoming increasingly more expensive and challenging the affordability and sustainability of drug programmes around the world. When new drugs are evaluated, health technology assessment organisations rely on clinical trials to inform funding decisions. However, clinical trials are not able to assess overall survival and generalises evidence in a real-world setting. As a result, policy makers have little information on whether drug funding decisions based on clinical trials ultimately yield the outcomes and value for money that might be expected.

Objective The Canadian Real-world Evidence for Value of Cancer Drugs (CanREValue) collaboration, consisting of researchers, recommendation-makers, decision makers, payers, patients and caregivers, are developing and testing a framework for Canadian provinces to generate and use real-world evidence (RWE) for cancer drug funding in a consistent and integrated manner.

Strategy The CanREValue collaboration has established five formal working groups (WGs) to focus on specific processes in the generation and use of RWE for cancer drug funding decisions in Canada. The different RWE WGs are: (1) Planning and Drug Selection; (2) Methods; (3) Data; (4) Reassessment and Uptake; (5) Engagement. These WGs are acting collaboratively to develop a framework for RWE evaluation, validate the framework through the multiprovince RWE projects and help to integrate the final RWE framework into the Canadian healthcare system. Outcomes The framework will enable the reassessment of cancer drugs, refinement of funding recommendations and use of novel funding mechanisms by decision-makers/ payers across Canada to ensure the healthcare system is providing clinical benefits and value for money.

\section{BACKGROUND}

New therapies for cancer are becoming increasingly more expensive, challenging the affordability and sustainability of public drug programmes in Canada and around the world. ${ }^{1-3}$ While the cost increases of new cancer therapies are evident, the benefits are not always clear. In the Canadian cancer drug funding process, after receiving regulatory approval from Health Canada, organisations such as the pan-Canadian Oncology Drug Review (pCODR) at the Canadian Agency of Drugs and Technologies in Health $(\mathrm{CADTH})$ and the Institut national d'excellence en santé et services sociaux (INESSS) in Quebec evaluate new cancer drugs and make funding recommendations to federal and provincial public payers. However, there is no policy infrastructure or framework in place for postfunding reassessment of cancer drugs, leaving payers and policy decisionmakers with limited opportunity to incorporate more mature evidence into funding decisions or to renegotiate prices with manufacturers based on evolving evidence and expanded use.

Traditionally, cancer drug funding decisions have been informed by randomised controlled trials (RCTs) ${ }^{45}$ but there can be practical and ethical challenges that make it difficult to conduct subsequent RCTs. ${ }^{6} 7$ Patients in trials can differ significantly from patients in practice and the results of RCTs can be different from real-world studies, ${ }^{8} 9$ limiting the generalisability of RCT evidence to a real-world setting. ${ }^{70}$ In recognition of these limitations and potential values of realworld evidence (RWE), the Canadian Realworld Evidence for Value of Cancer Drugs (CanREValue) collaboration was established. The purpose of this paper is to describe the CanREValue collaboration and its work toward integrating RWE into cancer drug funding decision-making in Canada. 


\section{WHAT IS RWE AND WHY IS IT NEEDED?}

RWE, generated through the analysis of real-world data (RWD), can provide direct and generalisable evidence on the comparative effectiveness, safety and cost-effectiveness of cancer drugs in clinical practice. RWD is generally defined as data collected in a non-RCT setting, and includes information typically found in a variety of sources such as electronic health records, disease registries and administrative databases. ${ }^{11-13}$ RWE is more prone to data bias and confounding than evidence provided through an RCT due to lack of randomisation. However, RWE has greater external validity by including patients who are often not represented in RCTs, such as older adults, smokers and patients with comorbidities. ${ }^{14}$ In addition, RWE has the advantage of generating evidence in a less expensive manner than RCTs. ${ }^{15}$

Multiple stakeholders including decision-makers/ payers, health technology assessment (HTA) agencies (pCODR/CADTH and INESSS), clinicians and the pharmaceutical industry have an interest in using RWE. From the decision-makers/payers' perspectives, comparative RWE studies of cancer drugs against funded alternatives are relevant to policy-making and can be used to support system sustainability. ${ }^{11}$ As population-level analyses, RWE can provide valuable insights on drugs for rare indications. The results of RWE studies can also improve payers' ability to negotiate with industry, including establishing postmarket commitments and requirements, conditional approvals or reimbursement and risk-sharing agreements. ${ }^{17} 18$ Recommendation-makers, such as HTA agencies, can also use comparative RWE evidence to reassess their past recommendations and provide updated guidance to decision makers/payers. ${ }^{19}$ The pharmaceutical industry uses RWE across all phases of the drug development lifecycle, ${ }^{20}$ from identification of patients with rare diseases to assessment of off-label efficacy. ${ }^{21} \mathrm{RWE}$ is also becoming increasingly used to conduct comparative effectiveness studies among treatment options that have not been compared directly in RCTs, which can help clinicians in their clinical decision-making. ${ }^{22-24}$

\section{ROLE OF CANREVALUE COLLABORATION}

While the potential value of RWE is well established in oncology research, technical and methodological challenges exist in its generation and use by different stakeholders. For example, the confluence of large datasets of uncertain quality, inappropriate data analyses and poor research design can produce results that are far from mirroring the 'real world'. ${ }^{25}$ Moreover, there is no consensus on how to incorporate RWE into funding decisions, which limits the use of RWE to inform decisionmaking for payers and decision-makers. The CanREValue collaboration aims to address these challenges and establish a framework for Canadian provinces regarding the generation and use of RWE for cancer drug funding decision making. The development and implementation of this framework will result in a process for evidence-based reassessment of recommendations on cancer drugs made by Canadian HTA organisations and mechanisms for the reconsideration of prior funding decisions and/or renegotiations of drug price. Ultimately, these recommendations, if implemented, will help support health system sustainability in Canada and potentially elsewhere.

The CanREValue collaboration was made possible through funding from a Partnerships for Health System Improvement grant awarded by the Canadian Institutes of Health Research and through partnerships with the Canadian Centre for Applied Research in Cancer Control and Cancer Care Ontario. Key stakeholders in the CanREValue collaboration include applied researchers, Health Canada, provincial ministries and departments of health, HTA organisations, provincial cancer agencies, the Canadian Association of Provincial Cancer Agencies, the pan-Canadian Pharmaceutical Alliance, the Patented Medicine Prices Review Board, clinicians, and patient and family representatives (online supplementary table S1). Industry is also a key player in the drug funding process and will be consulted throughout the framework development.

The CanREValue collaboration is a 4-year project. In the first year, an environmental scan and qualitative study on the use of RWE were conducted. The purpose of the environmental scan was to gain an understanding of the current state of RWE with respect to healthcare decisionmaking. In the qualitative study, stakeholders were interviewed to gain an understanding of their perspective on the development and implementation of a framework for the incorporation and use of RWE. The findings of these studies will be published separately. In subsequent years, the framework will be revised through RWE evaluation test cases, additional qualitative interviews and stakeholder consultations.

\section{CANREVALUE WORKING GROUPS}

The CanREValue collaboration has established five formal working groups (WGs) to focus on specific processes in the generation and use of RWE for cancer drug funding decisions in Canada (figure 1). The different RWE WGs are: (1) Planning and Drug Selection; (2) Methods; (3) Data; (4) Reassessment and Uptake; (5) Engagement (see table 1 for a summary of each WG). These WGs act collaboratively to develop a working framework; they will validate the framework through the multiprovince RWE projects, and will help integrate the final RWE framework into the Canadian healthcare system. Each WG meets to discuss and build consensus, using modified Delphi methods, ${ }^{26}{ }^{27}$ on issues related to the framework development. All WGs meet in-person once a year to share updates within and across WGs and to incorporate their findings into the overall framework.

\section{RWE Planning and Drug Selection WG}

With the development of an RWE framework, there are policy issues that need to be resolved. These include 


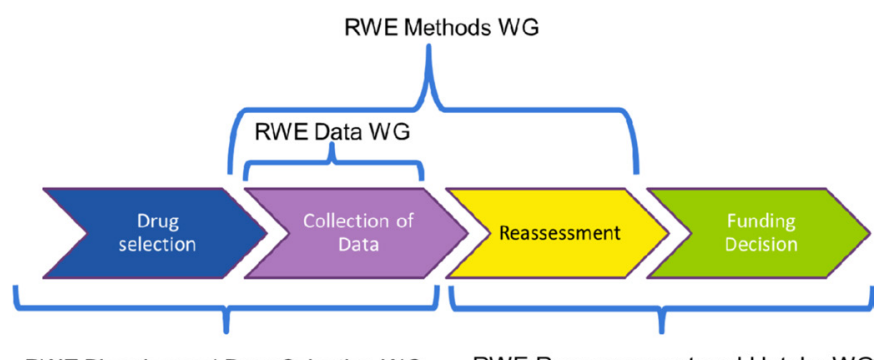

RWE Planning and Drug Selection WG

RWE Reassessment and Uptake WG

Stakeholder Engagement WG

Figure 1 The Canadian Real-world Evidence Value of Cancer Drugs project real-world evidence (RWE) working groups (WGs) and their roles within the development of the framework.

building consensus among payers and decision-makers regarding identification criteria of potentially policyrelevant RWE evaluations, developing policy infrastructure for the RWE study selection process and making recommendations for conducting RWE evaluations. The Planning and Drug Selection WG was established to develop criteria to identify potential drug indication candidates and select high-priority study questions for real-world evaluation. The focus of the Planning and Drug Selection WG is on identifying uncertainties at the time of drug funding that may be resolvable through the generation of RWE, along with considerations that should be factored into priority-setting among a basket of drug candidates. In addition, this WG will provide recommendations on potential infrastructure for conducting RWE studies.

The Planning and Drug Selection WG consists of numerous stakeholders involved in the cancer drug funding process including Health Canada, provincial health ministries/departments, provincial cancer agencies, HTA organisations, clinicians, patients and researchers. A widespread representation of key stakeholders is important for this WG to ensure that the needs and interests of a wide range of potential framework users are incorporated. Deliverables for the Planning and Drug Selection WG include a set of recommendations for selection criteria, prioritisation processes and infrastructure for RWE evaluations.

\section{RWE Data WG}

The CanREValue collaboration will focus on the generation of RWE using RWD collected from existing population-level administrative health databases, such as cancer registries, hospital records and insurance claims. Unfortunately, administrative healthcare data are often collected for routine operations and funding purposes and not research purposes. Thus, some elements required for research may not be present. Understanding the availability and usability of the existing data across provinces is important to address potential limitations upfront and to conduct meaningful RWE studies in the Canadian context.
The main tasks of the Data WG are to determine data availability and accessibility in each jurisdiction, and potential health or economic outcomes that can be measured from available data sources, such as survival, adverse events, patient-reported outcomes, resource utilisation and costs. In order to develop a framework that is applicable at a pan-Canadian level, there needs to be a strategy to identify and harmonise data elements from each province. Data experts from all 10 Canadian provinces are working together to explore the current data holdings and availability. These data experts will also be involved in conducting test case analyses as part of the framework development.

The anticipated deliverables for the Data WG are a list of strategies that can be used to identify, access and harmonise the data elements required to undertake RWE projects in each province and a report on provincial data availability and accessibility for cancer-specific RWE evaluations in Canada.

\section{RWE Methods WG}

The CanREValue collaboration will focus on conducting robust comparative observational studies, using clinically relevant cancer drugs/regimens currently administered in Canada. RWE is often generated from observational data, which makes it susceptible to bias that may hinder interpretation and use of the findings ${ }^{16}$ by decisionmakers and payers. ${ }^{1628}$ Therefore, to generate comparative RWE for policy decision-making, appropriate analytical approaches need to be selected, with careful consideration of issues related to choice of research question, study design and research methodology in light of available data. ${ }^{29}$ The goal of the Methods WG is to recommend appropriate statistical methods to be used to conduct robust RWE evaluations and ensure that the findings can provide meaningful information for payers and decision-makers.

The Methods WG consists of researchers with expertise in HTA, health services research, statistical analysis and health economics. The Methods WG will explore a variety of analytical strategies and the strengths and the limitations of different methods to address potential bias and confounders when estimating key outcomes, including safety, effectiveness and cost-effectiveness. The WG will then make recommendations on how to apply these methods to undertake RWE evaluations with support from the Data WG during the test case analyses.

The deliverables for the Methods WG include recommendations on appropriate statistical methods to conduct robust RWE evaluations using administrative health databases and publications about the conduct of RWE evaluations.

\section{RWE Reassessment and Uptake WG}

There can be uncertainties in the initial HTA of the benefits of a new drug due to limitations in the available evidence, such as a small sample size or a non-randomised study. ${ }^{30}$ Therefore, there is a need to develop a mechanism 
Table 1 The summary table for each working group (WG)

\begin{tabular}{|c|c|c|c|}
\hline Working groups & Goals & Key issues to address & Expected deliverables \\
\hline $\begin{array}{l}\text { Planning and Drug } \\
\text { Selection WG }\end{array}$ & $\begin{array}{l}\text { To recommend a selection } \\
\text { process and criteria for } \\
\text { prioritising RWE evaluations }\end{array}$ & $\begin{array}{l}\text { Potential markers or signals } \\
\text { for emerging drugs. } \\
\text { Policy issue around resource } \\
\text { allocation and infrastructure } \\
\text { for conducting RWE } \\
\text { evaluations. }\end{array}$ & $\begin{array}{l}\text { Criteria for selecting and } \\
\text { prioritising RWE evaluation. } \\
\text { Policy framework for drug } \\
\text { selection for RWE evaluation. } \\
\text { Prioritisation process for } \\
\text { drug selection. }\end{array}$ \\
\hline Methods WG & $\begin{array}{l}\text { To recommend statistical } \\
\text { methods to analyse real world } \\
\text { data }\end{array}$ & $\begin{array}{l}\text { Strengths and limitations of } \\
\text { different analytical strategies. } \\
\text { Appropriate methods } \\
\text { for measuring and } \\
\text { estimating key variables } \\
\text { (safety, effectiveness and } \\
\text { cost-effectiveness). }\end{array}$ & $\begin{array}{l}\text { Recommendations on } \\
\text { statistical methods to } \\
\text { conduct RWE evaluations. } \\
\text { Papers on conducting RWE } \\
\text { evaluations. }\end{array}$ \\
\hline $\begin{array}{l}\text { Reassessment and Uptake } \\
\text { WG }\end{array}$ & $\begin{array}{l}\text { To make recommendation on } \\
\text { a reassessment process and } \\
\text { strategies for incorporating } \\
\text { RWE results into policy } \\
\text { decisions }\end{array}$ & $\begin{array}{l}\text { How reassessment will be } \\
\text { initiated and conducted. } \\
\text { Funding decision revision. }\end{array}$ & $\begin{array}{l}\text { Development of policy } \\
\text { framework for reassessment } \\
\text { and funding decision. } \\
\text { Development of } \\
\text { reassessment process. } \\
\text { Recommendation on } \\
\text { strategies for the uptake of } \\
\text { the RWE framework. }\end{array}$ \\
\hline Engagement WG & $\begin{array}{l}\text { To ensure that all key } \\
\text { stakeholders are given a } \\
\text { voice in the development and } \\
\text { implementation of the RWE } \\
\text { framework }\end{array}$ & $\begin{array}{l}\text { Engagement of patient } \\
\text { groups, clinician groups, } \\
\text { industry and payers. }\end{array}$ & $\begin{array}{l}\text { Collect and provide feedback } \\
\text { from patient groups, clinician } \\
\text { groups, industry and payers. }\end{array}$ \\
\hline
\end{tabular}

RWE, real-world evidence.

to reassess the benefits of some drugs based on evidence generated after initial HTA recommendation. This reassessment may allow revised funding decisions and pricing discussion based on internationally evolving RWE. The purpose of the Reassessment and Uptake WG is to make recommendations on a formal reassessment process and propose strategies for incorporating RWE findings into policy decisions. Building on experience and expertise in HTA and policy decision-making, their work will focus on developing a reassessment framework to conduct a structured, evidence-based critical appraisal of the clinical, social, ethical and economic effects of a technology currently used in the healthcare system. This WG will develop a process that outlines how reassessment will be initiated and by whom, along with how reassessment will be conducted, including criteria for reassessment, recommendation outcomes, stakeholders to be involved and evidence required to maintain or revise the initial funding recommendations. These tasks will increase transparency and confidence in reassessment, as well as the utilisation of RWE evaluations.

Similar to the Planning and Drug Selection WG, the Reassessment and Uptake WG consists of individuals from Health Canada, provincial health ministries/departments, provincial cancer agencies, HTA organisations, cancer drug recommendation and decision makers, clinicians, patients and researchers. Deliverables for the Reassessment and Uptake WG include a systematic 
process for reassessing cancer drugs that are currently funded. In addition, the WG will provide recommendations to address issues relating to the uptake of the framework including how decision-makers/payers, HTA and industry can work together to initiate reassessment and renegotiation based on the results of an RWE evaluation.

\section{Engagement WG}

As previously noted, the development and implementation of the RWE framework requires comprehensive collaboration and discussion among the various key stakeholders. The purpose of the Engagement WG is to ensure that all key stakeholders are given a voice in the development and implementation of the RWE framework. The Engagement WG consists of individuals with perspectives from HTA organisations, provincial cancer agencies, patients, clinicians and researchers. These individuals have a network of connections, expertise and experiences in communicating with various stakeholders.

The Engagement WG will focus on developing approaches to engage with industry, patient groups, clinicians and payers throughout the project. This will be an iterative consultation process through formal and informal discussion sessions, as well as elicitation and incorporation of stakeholder feedback on preliminary drafts of reports and recommendations from the other WGs. This will ensure that the framework development is an inclusive process, which in turn will help to ensure that the final framework recommendations are supported and adopted.

\section{CONCLUSION}

For the successful development and uptake of an RWE framework, we recognise that robust evidence should be generated through RWE evaluations. At the same time, a shared understanding among stakeholders in the cancer drug funding landscape needs to be developed and agreed on. To this end, the CanREValue collaboration is bringing together key stakeholders from across the system and country to develop clear guidance, methods and processes for how RWE could be generated and used to support cancer drug funding decision-making in Canada. The diversity of the WG members and their expertise ensure that key issues in the generation and use of RWE are discussed and recommendations and decisions are made using the best evidence possible.

Cancer drug funding decisions are becoming progressively more difficult because of the increasing numbers of drugs, their escalating costs, ${ }^{31}$ and the uncertainty of their clinical benefit in the real world. ${ }^{32}$ The assessment of realworld performance of cancer drugs may provide opportunities to introduce new funding mechanisms such as conditional listing, outcomes-based managed entry or value-based pricing. RWE evidence may also enable the renegotiation of drug prices, which may free up funds to allow more drugs to be funded. Ultimately, the CanREValue collaboration is working to build consistency in the use of RWE at a national level, which will lead to a robust pan-Canadian system supporting sustainability, value for money and improved patient care.

Author affiliations

${ }^{1}$ Odette Cancer Centre, Sunnybrook Health Sciences Centre, Toronto, Ontario, Canada

${ }^{2}$ Institute of Health Policy, Management and Evaluation, University of Toronto, Toronto, Ontario, Canada

${ }^{3}$ Canadian Centre for Applied Research in Cancer Control, Toronto, Ontario, Canada ${ }^{4}$ Department of Oncology, Provincial Drug Reimbursement Programs, Cancer Care Ontario, Toronto, Ontario, Canada

${ }^{5}$ Department of Oncology, McMaster University, Hamilton, Ontario, Canada

${ }^{6}$ Institute for Mental Health Policy Research, Centre for Addiction and Mental Health Toronto, Ontario, Canada

${ }^{7}$ Provincial Drug Reimbursement Programs, pCODR/CADTH, Toronto, Ontario, Canada

${ }^{8}$ Department of Public Health Sciences, University of California Davis, Davis, California, USA

${ }^{9}$ Women's College Research Institute, Women's College Hospital, Toronto, Ontario, Canada

${ }^{10}$ Centre for excellence in Economic Analysis Research (CLEAR), St. Michael's Hospital, Li Ka Shing Knowledge Institute, Toronto, Ontario, Canada

${ }^{11}$ Health Intervention and Technology Assessment Program, Royal Thai Government Ministry of Public Health, Nontaburi, Thailand

Correction notice This article has been corrected since it was published. The last name for the author, Claire de Oliveira, has been amended.

Twitter Claire de Oliveira @clairede0

Contributors $\mathrm{KC}, \mathrm{WI}, \mathrm{BE}, \mathrm{CdO}, \mathrm{AC}, \mathrm{SG}, \mathrm{JH}$ and JB devised the project, the main conceptual ideas and proof outline. SN wrote the manuscript with critical feedback from all authors (KC, WI, BE, CdO, AC, SG, JH, REM, WFD, JB, MT).

Funding This work was supported by the Canadian Institutes of Health Research (Grant \#HRC-154126). This study was supported by the Canadian Centre for Applied Research in Cancer Control (ARCC). ARCC is funded by the Canadian Cancer Society Grant \# 2015-703549

Competing interests None declared.

Patient consent for publication Not required.

Provenance and peer review Not commissioned; externally peer reviewed.

Open access This is an open access article distributed in accordance with the Creative Commons Attribution Non Commercial (CC BY-NC 4.0) license, which permits others to distribute, remix, adapt, build upon this work non-commercially, and license their derivative works on different terms, provided the original work is properly cited, appropriate credit is given, any changes made indicated, and the use is non-commercial. See: http://creativecommons.org/licenses/by-nc/4.0/.

ORCID iD

Seungree Nam http://orcid.org/0000-0003-1705-3849

\section{REFERENCES}

1 Anas R, Gavura S, McLeod R, et al. Ontario's approach to tackling drug funding sustainability. J Clin Oncol 2016;34:38.

2 Canadian Institute for Health Information. National health expenditure trends, 1975 to 2017. Ottawa, ON, 2017.

3 Cancer Quality Council of Ontario. Sustainable cancer care: strengthening the system for future generation. Toronto, ON, 2016.

4 Murphy G. Use of real-world evidence in single-drug assessments. Ottawa, ON: CADTH, 2018.

5 Canadian Partnership Against Cancer and pCODR. How cancer drug funding decisions are made, 2013.

6 Howie LJ, Peppercorn JM. The ethics of clinical trials for cancer therapy. N C Med J 2014;75:270-3.

7 Logviss K, Krievins D, Purvina S. Characteristics of clinical trials in rare vs. common diseases: a register-based Latvian study. PLOS One 2018;13:e0194494.

8 Khor S, Beca J, Krahn M, et al. Real world costs and costeffectiveness of rituximab for diffuse large B-cell lymphoma patients: a population-based analysis. BMC Cancer 2014;14:586. 
9 Lakdawalla DN, Shafrin J, Hou N, et al. Predicting real-world effectiveness of cancer therapies using overall survival and progression-free survival from clinical trials: empirical evidence for the ASCO value framework. Value Health 2017;20:866-75.

10 Lewis JRR, Lipworth WL, Kerridge IH, et al. The economic evaluation of personalised oncology medicines: ethical challenges. Med J Aust 2013;199:471-3.

11 Food and Drug Administration. Framework for FDA's real-world evidence program, 2018.

12 Garrison LP, Neumann PJ, Erickson P, et al. Using real-world data for coverage and payment decisions: the ISPOR real-world data Task force report. Value Health 2007;10:326-35.

13 Makady A, de Boer A, Hillege $\mathrm{H}$, et al. What is real-world data? A review of definitions based on literature and Stakeholder interviews. Value Health 2017;20:858-65.

14 Suvarna V. Real world evidence (RWE) - Are we (RWE) ready? Perspect Clin Res 2018;9:61.

15 Katkade VB, Sanders KN, Zou KH. Real world data: an opportunity to supplement existing evidence for the use of long-established medicines in health care decision making. J Multidiscip Healthc 2018;11:295-304.

16 Hampson Get al. Real world evidence for coverage decisions: opportunities and challenges, 2018.

17 Andersson SW, Kyhlstedt M. Deriving more value from RWE to ensure timely access of medicines by patients. J Comp Eff Res 2017;6:391-5.

18 The Nertwork for Excellence in Health Innovation. Real world evidence: a new era for health care innovation, 2015

19 Makady A, Ham RT, de Boer A, et al. Policies for use of real-world data in health technology assessment $(\mathrm{HTa})$ : a comparative study of six HTa agencies. Value Health 2017;20:520-32.

20 Hughes B, Kessler M, McDonell A. Breaking new ground with RWE: How some pharmacos are poised to realize a $\$ 1$ billion opportunity 2016.

21 Gill JLet al. RWE in Eurpoe paper IV: engaging pharma in the RWE roadmap 2017.
22 Heng DY, Signorovitch J, Swallow E, et al. Comparative effectiveness of second-line targeted therapies for metastatic renal cell carcinoma: a systematic review and meta-analysis of real-world observational studies. PLoS One 2014;9:e114264.

23 Laliberté F, Cloutier M, Nelson WW, et al. Real-World comparative effectiveness and safety of rivaroxaban and warfarin in nonvalvular atrial fibrillation patients. Curr Med Res Opin 2014;30:1317-25.

24 Makady A, Stegenga H, Ciaglia A, et al. Practical implications of using real-world evidence (RWE) in comparative effectiveness research: learnings from IMI-GetReal. J Comp Eff Res 2017;6:485-90.

25 Sherman RE, Anderson SA, Dal Pan GJ, et al. Real-World evidence - what is it and what can it tell us? N Engl J Med 2016;375:2293-7.

26 Hsu C, Sanford B. The Delphi technique: making sense of consensus. Practical Assessment, Research \& Evaluation 2007;12:1-8.

27 Loblaw DA, Prestrud AA, Somerfield MR, et al. American Society of clinical oncology clinical practice guidelines: formal systematic Review-Based consensus methodology. JCO 2012;30:3136-40.

28 Food and Drug Administration. Use of real-world evidence to support regulatory decision-making for medical devices 2017.

29 Booth CM, Karim S, Mackillop WJ. Real-World data: towards achieving the achievable in cancer care. Nat Rev Clin Oncol 2019;16:312-25.

30 Woolacott N, Corbett M, Jones-Diette J, et al. Methodologica challenges for the evaluation of clinical effectiveness in the context of accelerated regulatory approval: an overview. J Clin Epidemiol 2017;90: :108-18.10.1016/j.jclinepi.2017.07.002

31 de Oliveira C, Weir S, Rangrej J, et al. The economic burden of cancer care in Canada: a population-based cost study. CMAJ Open 2018;6:E1-10.

32 Baik CS, Rubin EH, Forde PM, et al. Immuno-oncology clinical trial design: limitations, challenges, and opportunities. Clin Cancer Res 2017;23:4992-5002. 